EDITOR'S CHOICE

\title{
Compliance with a comprehensive warm-up programme to prevent injuries in youth football
}

\author{
Torbjørn Soligard, ${ }^{1}$ Agnethe Nilstad, ${ }^{1}$ Kathrin Steffen, ${ }^{1}$ Grethe Myklebust, Ingar Holme, \\ Jiri Dvorak, ${ }^{2}$ Roald Bahr, ${ }^{1}$ Thor Einar Andersen ${ }^{1}$
}

${ }^{1}$ Oslo Sports Trauma Research Center,Norwegian School of Sport Sciences, Ullevaal Stadion, Oslo, Norway 2FIFA Medical Assessment and Research Centre, Schulthess Clinic, Zürich, Switzerland

\section{Correspondence to} Dr Torbjørn Soligard,

Norwegian School of Sport Sciences, Oslo Sports Trauma Research Center, PO Box 4014, Ullevaal Stadion, 0806 Oslo, Norway;

torbjorn.soligard@nih.no

Accepted 5 April 2010 Published Online First 15 June 2010

\section{ABSTRACT}

Background Participants' compliance, attitudes and beliefs have the potential to influence the efficacy of an intervention greatly.

Objective To characterise team and player compliance with a comprehensive injury prevention warm-up programme for football (The 11+), and to assess attitudes towards injury prevention among coaches and their association with compliance and injury risk.

Study Design A prospective cohort study and retrospective survey based on a cluster-randomised controlled trial with teams as the unit of randomisation. Methods Compliance, exposure and injuries were registered prospectively in 65 of 125 football teams (1055 of 1892 female Norwegian players aged 13-17 years and 65 of 125 coaches) throughout one football season (March-October 2007). Standardised telephone interviews were conducted to assess coaches' attitudes towards injury prevention.

Results Teams completed the injury prevention programme in 77\% (mean 1.3 sessions per week) of all training and match sessions, and players in 79\% (mean 0.8 sessions per week) of the sessions they attended. Compared with players with intermediate compliance, players with high compliance with the programme had a $35 \%$ lower risk of all injuries (RR 0.65, 95\% Cl 0.46 to $0.91, p=0.011$ ). Coaches who had previously utilised injury prevention training coached teams with a $46 \%$ lower risk of injury (OR $0.54,95 \% \mathrm{Cl} 0.33$ to 0.87 , $\mathrm{p}=0.011$ ).

Conclusions Compliance with the injury prevention programme was high, and players with high compliance had significantly lower injury risk than players with intermediate compliance. Positive attitudes towards injury prevention correlated with high compliance and lower injury risk.

Frameworks have been outlined to describe the systematic approach needed to build an evidence base for the prevention of sports injuries. ${ }^{1-3}$ The effectiveness of an injury prevention programme depends, among other things, on uptake of the intervention among participants, that is, compliance. Therefore, to prevent injuries, it is crucial to understand the factors that influence athletes, coaches and sports administrators to accept, adopt and comply with the elements of the intervention. $^{2} 3$

Documentation of participant compliance is often incomplete in studies examining the effectiveness of injury prevention protocols in team sports; the documentation of participant compliance is inconsistent. Whereas a number of studies have neglected compliance altogether, ${ }^{4-12}$ some have noted the importance of compliance, but not reported it. ${ }^{13-18}$ Others have reported compliance, but not linked it to an injury prevention effect estimate. ${ }^{19-26}$ Finally, some studies have linked compliance to an effectiveness estimate. ${ }^{27-32}$ We thus have limited data on the relationship between compliance and effectiveness.

Furthermore, when injury prevention measures are embedded into team training sessions, the compliance of the team is likely to depend greatly on the motivation, choices and actions of the head coach. We therefore determined to what degree an intervention is accepted and adopted by coaches. Recording individual participation, on the other hand, reveals the rate of uptake and actual usage of the intervention for each player. Recording team and player compliance together will provide detailed data on the overall compliance with the intervention (figure 1).

The primary aim of this study was to characterise the compliance of youth teams and players using an injury prevention training programme and to examine whether high compliance correlated with lower injury risk. We also wanted to identify coaches' attitudes towards injury prevention training and to examine whether their attitudes were associated with the compliance or the risk of injury within their teams.

\section{METHODS}

This study is based on data from a cluster-randomised controlled trial on young female footballers (soccer players) examining the injury preventive effect of a comprehensive warm-up programme (The 11+). The design, intervention programme and main results have been reported. ${ }^{33}$

\section{Participants}

Of the 181 teams organised in the girls' 15 and 16-year divisions in the south, east and middle regional districts of the Norwegian Football Association, 65 out of 125 teams entering the study were randomly assigned to the intervention group and formed the basis for the present paper (figure 2). To be included, teams had to carry out at least two training sessions per week, in addition to matches played. The competitive season lasted from the end of April until midOctober 2007, interrupted by a 7-week summer break. All teams were also followed for 2 months of preseason training (March-April). The recording of compliance included all the teams $(n=65)$ in the intervention group, and the investigation online under the BMJ Journals unlocked scheme, see http:// bjsm.bmj.com/info/unlocked.dtl 


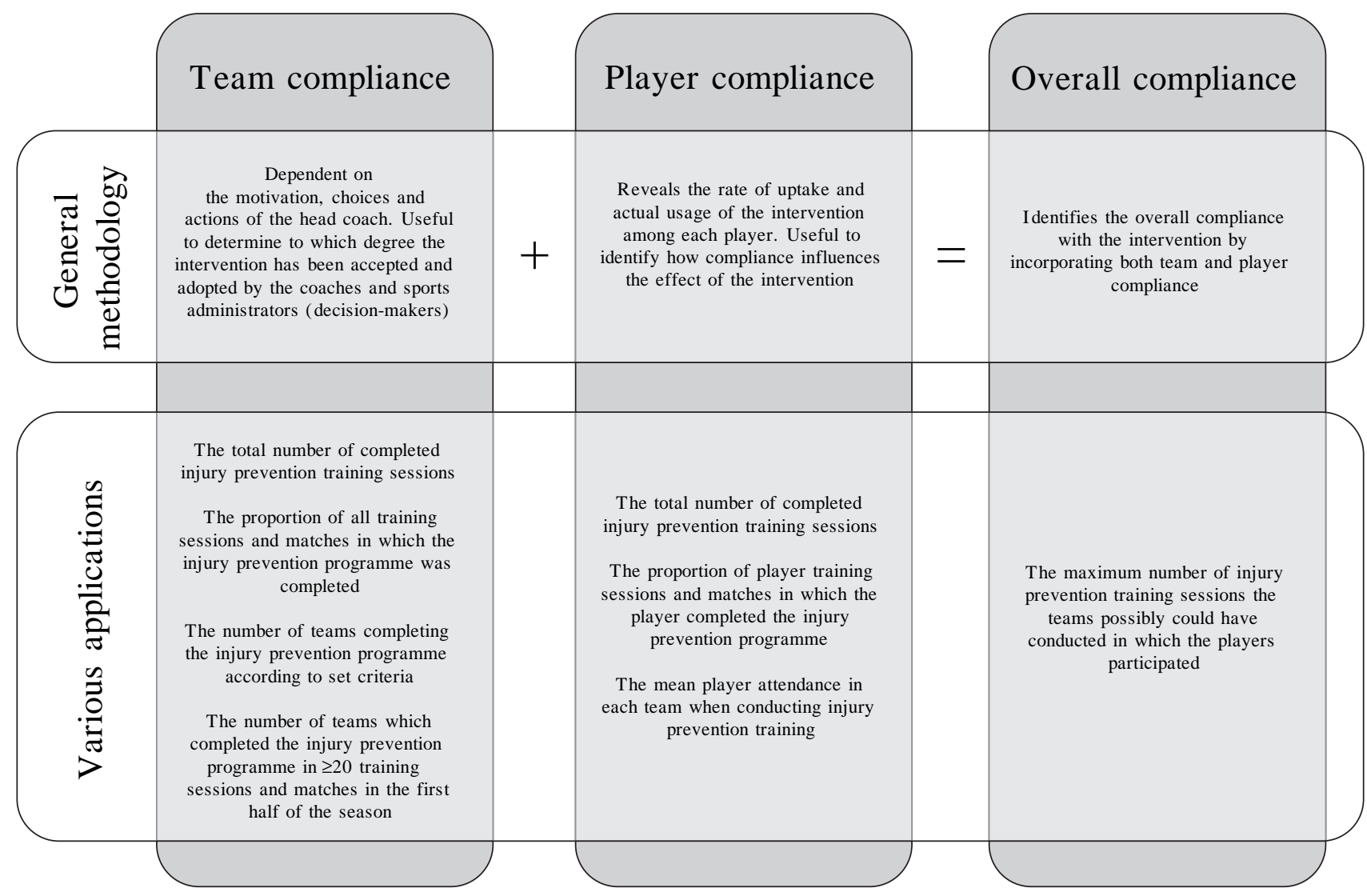

Figure 1 The distinction between compliance among teams and players, and definitions of compliance used in this study.

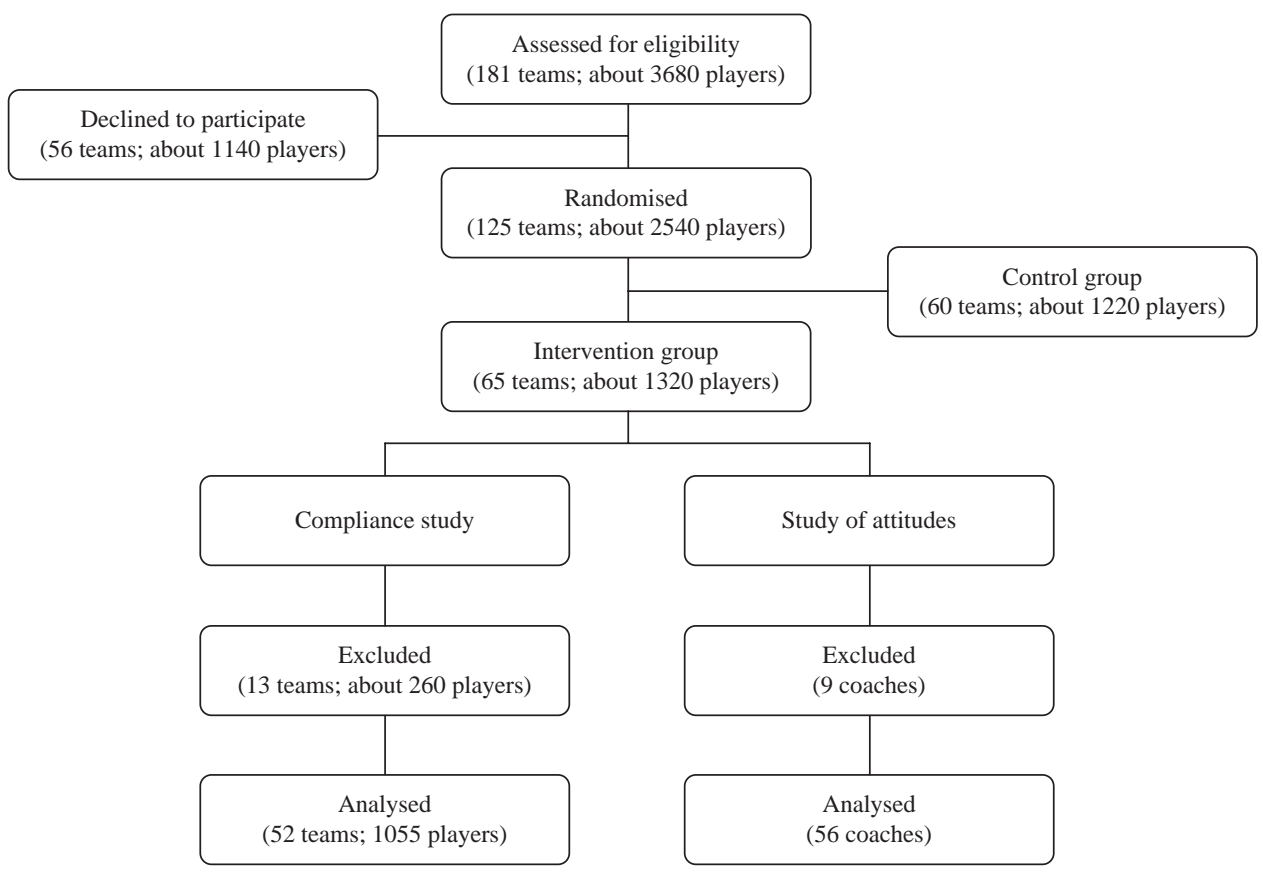

Figure 2 Flow of team clusters and players throughout the study.

of attitudes and beliefs towards injury prevention included all the coaches $(n=65)$ of the intervention teams.

\section{Compliance recording and reporting}

The coaches reported injuries and individual player participation prospectively, as the number of minutes of exposure, for each training session and match on weekly registration forms throughout the study period. Furthermore, for each session the coaches quantitatively recorded whether the warm-up programme was carried out, as well as the participation of each player in the programme (yes/no). The registration forms were submitted by e-mail, mail, or fax to the Oslo 
Sports Trauma Research Center. Data on players who dropped out during the study period were included for the entire period of their participation. For comparison with results from previous studies compliance was defined and reported in multiple ways (figure 1).

\section{Injury recording}

One physical therapist and one medical student were given specific training on the protocols for injury classification and injury definitions (see Soligard et al) ${ }^{33}$ before the start of the injury recording period. These injury recorders called every injured player to assess detailed aspects of the injury based on a standardised injury questionnaire, ${ }^{34}$ and the players were in most cases reached within 4 weeks (range 1 day to 5 months) after the injury had occurred.

\section{Study of attitudes and beliefs towards injury prevention}

After the season, from mid-October to November, every coach in the intervention group was called to evaluate the complete warm-up programme and the exercises used, as well as to assess attitudes and beliefs towards injury prevention training in general. This retrospective study was based on a questionnaire designed by the authors, consisting of 28 closed and three open questions. The questionnaire was standardised using dichotomous or five-point Likert scale response alternatives in accordance with questionnaire design guidelines to ensure reliability and validity. ${ }^{35}$ All interviews were conducted by a physical therapist (AN).

\section{Statistical methods}

This report is based on an exploratory post hoc analysis of data from the intervention group in a randomised controlled trial. ${ }^{33}$ All statistical analyses were conducted using SPSS for Windows version 15.0 and STATA version 10.0. We used a Poisson regression model based on generalised estimating equations taking cluster effects into account as a per protocol analysis to compare the rate ratios (RR) of the risk of injury between teams as well as players (independent of club) stratified into tertiles of compliance according to the number of prevention sessions completed: low, intermediate and high. We used $\chi^{2}$ tests to compare categorical variables between these subgroups and one-way analysis of variance to compare continuous variables. To investigate the relation between the coaches' attitudes and compliance with the warm-up programme, logistic regression analyses were used with compliance as the dependent variable. Attitudes among coaches who represented teams with high compliance were compared with attitudes among coaches from low-compliance teams. The teams who completed both the intervention study and the study of attitudes were included in this analysis. To investigate the relation between the coaches' attitudes and their teams' injury risk, logistic regression analyses were used with injury risk as the dependent variable. The results are presented as OR with $95 \% \mathrm{CI}$ and $\mathrm{p}$ values. The summary measure of injury incidence (i) was calculated according to the formula $i=n / e$, where $n$ is the number of injuries during the study period and $e$ the sum of exposure time expressed in player hours of match, training or in total. Descriptive data for exposure, compliance with the warm-up programme, injury incidences and attitudes towards injury prevention training are presented as means with standard errors or $95 \%$ CI. RR are presented with $95 \%$ CI. Two tailed $p$ values of 0.05 or less were regarded as significant.

\section{RESULTS}

Of the 65 teams in the intervention group, 52 (1055 players) completed the season and thus the compliance study. Fifty-six coaches completed the study of attitudes and beliefs towards injury prevention training; 50 belonged to teams that completed the compliance study, whereas six belonged to teams that dropped out during the season (figure 2).

\section{Compliance of teams}

The 52 teams completed the injury prevention programme in 2279 (mean 44 22 sessions, range 11-104) out of 2957 training sessions and matches throughout the season (77\%), corresponding to 1.3 times per week. Of all the teams, $60 \%(n=31)$ completed the injury prevention programme two times per week or more in accordance with the recommendation. In all tertiles of compliance, the majority of the injury prevention sessions were conducted in the first half of the season (MarchJune). In this period the programme was completed in $82 \%$ of all sessions, whereas $75 \%$ of the teams $(n=39)$ completed the prevention programme in 20 or more sessions (table 1). In the second part of the season (August-October) the programme was completed in $58 \%$ of all sessions. The difference in compliance between the first and the second part of the season was particularly noticeable in the tertile with low compliance; these teams completed the injury prevention programme seven times more often in the first part of the season. In the second half of the season the teams in the lowest tertile completed the programme in $2.4 \pm 4.1$ sessions over a period of 11 weeks.

\section{Compliance of players}

The 1055 players completed the injury prevention programme in 28212 (mean $27 \pm 19$ sessions, range 0-95) out of 35589 sessions throughout the season $(79 \%)$, corresponding to 0.8 sessions per week. However, for each session the average number of players per team that participated in the injury prevention programme was 12.0 , corresponding to only $59 \%$ of all players on the roster (mean 20.3 per team). As the team compliance was $77 \%$, all the enrolled players therefore completed the injury prevention programme in $47 \%$ of the maximum number of sessions the teams possibly could have conducted.

The tertile of players with high compliance completed the injury prevention programme more than six times as often as players in the tertile with lowest compliance (table 1).

\section{Compliance and injury risk}

There was no difference in the risk of injury between teams with high, intermediate and low compliance (table 2). However, the risk of injury was 35\% ( $p=0.011)$ lower among players in the tertile with the highest compliance (mean 49.2 sessions per season, 1.5 sessions per week; range 33-95 sessions per season) compared with players in the intermediate tertile (mean 23.4 sessions per season, 0.7 sessions per week; range 15-32 sessions per season). In contrast, there was no significant reduction ( $p=0.13$ ) of injury risk between the intermediate tertile and the tertile with the lowest compliance (mean 7.7 sessions per season, 0.2 sessions per week; range 0-14 sessions). Furthermore, the risk of an acute injury was $39 \%(p=0.008)$ lower for players in the tertile with the highest compliance compared with players in the intermediate tertile, whereas a $35 \%$ reduction of injury risk compared with the tertile with the lowest compliance was not statistically significant $(p=0.09)$. 
Table 1 Team and player compliance with the injury prevention programme stratified into tertiles of compliance

\begin{tabular}{|c|c|c|c|c|c|c|c|c|}
\hline & Mean \pm SD & Range & Mean \pm SD & Range & Mean \pm SD & Range & Mean \pm SD & Range \\
\hline First half of the season & $43.4 \pm 9.2$ & $34-66$ & $28.3 \pm 6.2$ & $18-36$ & $18.7 \pm 7.0$ & $4-28$ & $30.1 \pm 12.6$ & $4-66$ \\
\hline Second half of the season & $19.8 \pm 7.8$ & $9-40$ & $13.1 \pm 6.0$ & $0-22$ & $2.4 \pm 4.1$ & $0-12$ & $11.8 \pm 9.4$ & $0-40$ \\
\hline The whole season & $68.6 \pm 14.8$ & 52-104 & $42.3 \pm 5.8$ & $30-52$ & $20.6 \pm 5.6$ & $11-28$ & $43.8 \pm 21.8$ & 11-104 \\
\hline
\end{tabular}

Values are mean numbers of injury prevention sessions completed in the different periods of the season, presented with SD and ranges.

Table 2 Injury risk among teams and players stratified into high, intermediate and low compliance

\begin{tabular}{|c|c|c|c|c|c|c|}
\hline & \multicolumn{3}{|l|}{ Teams } & \multicolumn{3}{|l|}{ Players } \\
\hline & Injury incidence & Rate ratio & $\mathrm{p}$ Value & Injury incidence & Rate ratio & p Value \\
\hline \multicolumn{7}{|l|}{ All injuries } \\
\hline Intermediate compliance & $3.7(2.8-4.7)$ & $0.84(0.59-1.78)$ & 0.30 & $4.0(3.0-5.0)$ & $0.65(0.46-0.91)$ & 0.011 \\
\hline Low compliance & $2.7(1.6-3.7)$ & $1.17(0.75-1.85)$ & 0.49 & $3.7(2.2-5.3)$ & $0.68(0.41-1.12)$ & 0.13 \\
\hline Intermediate compliance & $3.4(2.5-4.3)$ & $0.73(0.50-1.05)$ & 0.09 & $3.5(2.5-4.4)$ & $0.61(0.42-0.88)$ & 0.008 \\
\hline Low compliance & $2.3(1.3-3.3)$ & $1.06(0.65-1.74)$ & 0.81 & $3.3(1.8-4.7)$ & $0.65(0.39-1.08)$ & 0.09 \\
\hline
\end{tabular}

High compliance tertile is reference group.

\section{Coach attitudes, compliance and injury risk}

All the coaches $(n=56)$ expressed that including injury prevention training in the training programme is important; $80 \%$ $(n=45)$ stated that it is 'very important' and $20 \%(n=11)$ that it is 'important'. Regarding the perceived risk of sustaining an injury, $29 \%(n=16)$ of the coaches believed that their players were at high risk, $59 \%(n=33)$ believed that the risk of injury was intermediate and $13 \%(n=7)$ believed that the risk was low. However, $54 \%(n=30)$ of the coaches had never previously conducted injury prevention training. According to $75 \%(n=42)$ of the coaches, the media and profiled athletes largely influence their motivation to carry out injury prevention training. The majority of the coaches believed that the motivation of the coach is significant when trying to motivate young female football players to do injury prevention training $(95 \%, n=53)$.

Of the coaches from teams with high compliance, $94 \%$ $(n=16)$ believed that the players' motivation to complete the injury prevention programme was high, as opposed to $41 \%$ $(n=7)$ of the coaches from low-compliance teams. The probability of having low compliance with the injury prevention programme was $87 \%$ higher if the coach believed that the programme was too time-consuming (OR 0.13, 95\% CI 0.03 to $0.60, p=0.009)$. The opinion that this injury prevention programme did not include enough football-specific activities resulted in an $81 \%$ higher probability of low compliance with the programme (OR $0.19,95 \%$ CI 0.40 to $0.92, p=0.038$ ). Whether the coach had previously utilised injury prevention training in a similar group of players did not influence the compliance with the injury prevention programme (OR 0.60, 95\% CI 0.14 to $2.47, \mathrm{p}=0.47$ ).

There was no significant relationship between the injury risk of the teams and the overall attitude towards injury prevention training among their coaches $(p=0.33)$. However, compared with teams with coaches who had never undertaken injury prevention training before, teams with coaches who had used such training previously had $46 \%$ fewer injuries (OR 0.54, 95\% CI 0.33 to $0.87, \mathrm{p}=0.011$ ).

\section{DISCUSSION}

In this study, compliance was good; teams used the injury prevention programme in $77 \%$ of all training sessions and matches and players completed the programme in $79 \%$ of the sessions they attended. Also, the risk of overall and acute injuries was reduced by more than a third among players with high compliance compared with players with intermediate compliance.

\section{Compliance and risk of injury}

The players with high compliance completed twice as many injury prevention sessions as the players with intermediate compliance (1.5 vs 0.7 sessions per week). Interestingly, the preventive effect of The 11+ therefore increased with the rate of use, at least when conducted more than 1.5 times per week on average. No studies have similarly compared the risk of injury in players and teams with high, intermediate and low compliance with an intervention to prevent injuries. However, similar indications of exposure-response relationships have been found previously. ${ }^{28}$ Furthermore, a post hoc analysis showed that compared with the controls, ${ }^{33}$ players with high compliance experienced a $45 \%$ reduction in the overall risk of injury (data not shown), that is, an even greater effect than when compared with intervention players with intermediate and low compliance. 
Overall, the intervention players completed 0.8 injury prevention sessions each week on average, less than the recommendation of at least two sessions per week. However, they still experienced a $30-50 \%$ reduction in the risk of various injuries compared with the controls. This indicates that the injury prevention programme achieved the desired injury preventive effect.

In contrast to the findings among players, we found no significant differences in the overall or acute risk of injuries between teams with different levels of compliance. This is explained by the large variations in compliance among the players within each team; the players with high compliance had a sixfold higher use of the programme compared with the players with low compliance. These findings emphasise the inadequacy of recording compliance on a team basis only. The overall compliance is a product of the compliance among the teams and the player participation rate (figure 1). Although the compliance among teams and attending players was good, certain players in each team rarely took part in the team activities, despite being registered on the roster at the start of the season. Therefore, the whole group of enrolled players completed the injury prevention programme in $47 \%$ of the maximum number of sessions the teams possibly could have conducted.

It should be noted that the teams with low compliance reported three times lower exposure to football than the teams with high compliance, and four of 10 teams with low compliance did not report any injuries at all. Even though calculations of injury incidence take exposure into account, a minimum exposure is necessary to be at risk of injury. Moreover, coaches less thorough in conducting the injury prevention programme and recording compliance may also have been less likely to record injuries. If so, the injury incidence in the low compliance group may have been underestimated somewhat.

The programme was designed to prevent injuries. However, to make it attractive for coaches and players, The 11+ was specifically tailored to football players and we included elements of variation and progression in the exercise prescription. We also focused on organising streamlined and efficient $3 \mathrm{~h}$ educational meetings at baseline, at which the coaches were provided with a selection of material detailing the exercises. Although we gave a set of footballs to the teams that completed the collection of injuries and exposure, no incentives were provided to ensure high compliance by coaches and players other than telephone and e-mail contacts related to data collection. Indeed, the compliance rates among teams in the current study was higher than previously reported among teams, 182124272831 as well as among players. ${ }^{23} 2930$ In addition, our intervention period lasted longer than comparable interventions in other studies. Although compliance decreased from the first to the second half of the season, these findings may imply that a long-term intervention period is not synonymous with low motivation and compliance among the participants. Other factors, such as the content, the relevance, the availability and the perceived difficulty of the intervention may also play an important role.

\section{Attitudes towards injury prevention training}

Compliance with an intervention depends upon the motivation among the participants to perform a certain safety behaviour and that the barriers associated with the behaviour are limited. ${ }^{2}$ The strongest motivator for the coach was the expectation of fewer injuries. All coaches emphasised the importance of including injury prevention training in training, and the majority believed that the risk of injury among their players was high or intermediate. Nonetheless, more than half of the coaches had never previously conducted injury prevention training; this suggests that previous barriers associated with such training were too high.

The 11+ was completed in 20 min once the players were familiar with the programme. In addition to providing players with a solid warm-up, the programme included exercises aimed at improving strength, core stability, plyometrics and balance, components that presumably would be beneficial both in preventing injuries and enhancing performance. Nevertheless, time constraints were perceived as a barrier by many of the coaches. Moreover, if the coach held the opinion that the programme did not include enough football-specific activities, the probability of low compliance increased by $81 \%$. This indicates that content is important when implementing injury prevention measures in the sports community. The finding corresponds with theories proposing that when the barriers associated with a task are perceived as great, the task is less likely to be carried out. ${ }^{3637}$

All coaches believed that their attitudes towards injury prevention training influenced their players' motivation to perform the programme - they served as role models. Furthermore, the majority of coaches responded that the media and high-profile athletes influence the motivation to carry out injury prevention training. These findings are supported by well-founded theories suggesting that if people think their significant others want them to perform a behaviour, this results in a higher motivation and greater likelihood of action. ${ }^{36} 38$

Interestingly, injuries were half as likely in the teams of the coaches who previously in their coaching career had undertaken injury prevention training compared with teams of coaches who had not used such training. Previous experience with injury prevention training seems to improve the positive attitudes of coaches and may increase the implementation of The $11+$ in both training sessions and before matches.

\section{General methodological considerations}

A strength of the study is that the compliance was recorded both among teams and individual players, providing a detailed account of the acceptance of the intervention. In addition, the sample size of both players and coaches was large and the follow-up period was one complete football season. With respect to the coach interviews, the main objective was to identify the attitudes and beliefs towards injury prevention training among the coaches, but we also wanted to evaluate the warm-up programme and its exercises. As a consequence, the interviews were conducted after the season. However, the perceived risk of injury can easily influence the attitudes towards injury prevention training; ${ }^{36} 39$ thus, it would have been more appropriate to assess attitudes before the season and to evaluate the content of the programme after the season.

Regarding the relationship between coach attitudes, compliance and team injury risk, only coaches who completed the recording of compliance and injuries were included in the analyses. Although the most common barrier to study participation reported by coaches was the additional work of data recording and reporting, some teams may have dropped out due to low motivation towards the intervention programme. Therefore, coach attitudes to the programme may be less favourable than those reported by the study participants.

Except for a $3 \mathrm{~h}$ instructional course with the coaches and team captains in the preseason, the teams received no 
follow-up visits to refresh coaching skills or give players feedback on their performance. Throughout the season it was up to the coaches to make sure the exercises were performed properly with high quality. Although the programme proved to reduce the risk of several injury types, follow-up visits during the season could have proved helpful in ensuring the quality of the exercise performance and might possibly have resulted in an even higher preventive effect.

The coach of each team recorded the injuries, the exposure and the compliance. We did not monitor the validity and reliability of their recordings. In cases in which the registration form was not completed during or immediately after a training session or match the coach had to complete the registration form in a retrospective manner. However, recall bias is presumably small, because the majority of the coaches followed the protocol and submitted their registration forms on a weekly basis. Also, all teams were offered an incentive, provided they recorded all data throughout the study period. It is possible that coaches completed and submitted the registration forms merely to receive the reward, without ensuring the accuracy of the recorded data. This may have impaired the reliability of the submitted data.

\section{Implications}

Knowledge of factors that influence compliance with an intervention is still limited. This study is one of few that have aimed to identify these factors. The findings demonstrated that attitudes towards injury prevention training are associated with the rate of uptake of an intervention. Attitudes are developed from an early age. It may be important to implement injury prevention training as soon as children start participating in organised sports to make it a natural part of their training routines. It is also necessary to increase the understanding of the benefits of injury prevention among coaches in both youth and elite sports. Injury prevention training thus ought to be a core element of coach education and training programmes in football and other sports.

When recording and reporting compliance in team sports there should be a distinction between compliance among teams and among individual players. The compliance of a team is highly dependent on the motivation, choices and actions of the head coach. Recording individual participation, on the other hand, reveals the rate of uptake and actual usage of the intervention for each player. The recording of individual compliance is thus necessary to investigate how compliance influences the effect of an intervention and to identify possible exposure-response relationships. Recording team and player compliance together will provide detailed data on the overall compliance with the intervention (figure 1), and such methods should be applied in future research.

\section{CONCLUSION}

The compliance among players and teams with The 11+ injury prevention programme was high. The risk of overall and acute injuries was reduced by more than a third among players with high compliance. Positive coach attitudes correlated with high compliance and lower injury risk.

Acknowledgements The authors would like to thank the project assistants (Birgitte Lauersen, Ellen Blom, Olav Kristianslund and Tone Wigemyr), the coaches and the players who participated in this study.

Funding This study was supported by grants from the FIFA Medical Assessment and Research Centre. The Oslo Sports Trauma Research Center has been established at the Norwegian School of Sport Sciences through generous grants from the Royal
Norwegian Ministry of Culture and Church Affairs, the South-Eastern Norway Regional Health Authority, the Norwegian Olympic Committee and Confederation of Sport and Norsk Tipping AS.

\section{Competing interests None.}

Patient consent Obtained.

Ethics approval This study was conducted with the approval of the Regional Committee for Medical Research Ethics, South-Eastern Norway Regional Health Authority, Norway.

Provenance and peer review Not commissioned; externally peer reviewed.

\section{What is already known on this topic}

- The effectiveness of an injury prevention programme depends, among other things, on uptake of the intervention among participants, that is, compliance.

- Knowledge about the relationship between compliance and injury prevention effectiveness is limited.

\section{What this study adds}

- When embedding injury prevention into team training sessions, recording both team and player compliance is necessary to document overall compliance and exposureresponse relationships.

- Players with high compliance appear to benefit in terms of fewer injuries.

- Positive coach attitudes are associated with high compliance and lower injury risk.

\section{REFERENCES}

1. van Mechelen W, Hlobil H, Kemper HC. Incidence, severity, aetiology and prevention of sports injuries. A review of concepts. Sports Med 1992;14:82-99.

2. Finch C. A new framework for research leading to sports injury prevention. J Sci Med Sport 2006;9:3-9.

3. Finch $\mathbf{C F}$, Donaldson A. A sports setting matrix for understanding the implementation context for community sport. Br J Sports Med. Published Online First: 6 February 2009. doi: 10.1136/bjsm.2008.056069

4. Ekstrand J, Gillquist J, Liljedahl SO. Prevention of soccer injuries. Supervision by doctor and physiotherapist. Am J Sports Med 1983;11:116-20.

5. Tropp H, Askling C, Gillquist J. Prevention of ankle sprains. Am J Sports Med 1985;13:259-62.

6. Surve I, Schwellnus MP, Noakes T, et al. A fivefold reduction in the incidence of recurrent ankle sprains in soccer players using the Sport-Stirrup orthosis. Am J Sports Med 1994;22:601-6.

7. Heidt RS Jr, Sweeterman LM, Carlonas RL, et al. Avoidance of soccer injuries with preseason conditioning. Am J Sports Med 2000;28:659-62.

8. Junge A, Rosch D, Peterson $L$, et al. Prevention of soccer injuries: a prospective intervention study in youth amateur players. Am J Sports Med 2002;30:652-9.

9. Árnason Á, Engebretsen L, Bahr R. No effect of a video-based awareness program on the rate of soccer injuries. Am J Sports Med 2005;33:77-84.

10. Johnson U, Ekengren J, Andersen M. Injury prevention in Sweden: helping soccer players at risk. J Sport Exerc Psychol 2005;27:32-8.

11. Scase $\mathbf{E}$, Cook J, Makdissi M, et al. Teaching landing skills in elite junior Australian football: evaluation of an injury prevention strategy. Br J Sports Med 2006;40:834-8; discussion 838.

12. Mohammadi F. Comparison of three preventive methods to reduce the recurrence of ankle inversion sprains in male soccer players. Am J Sports Med 2007;35:922-6.

13. Caraffa A, Cerulli G, Projetti M, et al. Prevention of anterior cruciate ligament injuries in soccer. A prospective controlled study of proprioceptive training. Knee Surg Sports Traumatol Arthrosc 1996;4:19-21. 
14. Wedderkopp N, Kaltoft M, Holm R, et al. Comparison of two intervention programmes in young female players in European handball - with and without ankle disc. Scand J Med Sci Sports 2003;13:371-5.

15. Verhagen $\mathbf{E}$, van $\operatorname{der}$ Beek A, Twisk J, et al. The effect of a proprioceptive balance board training program for the prevention of ankle sprains: a prospective controlled trial. Am J Sports Med 2004;32:1385-93.

16. Emery CA, Cassidy JD, Klassen TP, et al. Effectiveness of a home-based balance-training program in reducing sports-related injuries among healthy adolescents: a cluster randomized controlled trial. Can Med Assoc J 2005;172:749-54

17. Mandelbaum BR, Silvers HJ, Watanabe DS, et al. Effectiveness of a neuromuscular and proprioceptive training program in preventing anterior cruciate ligament injuries in female athletes: 2-year follow-up. Am J Sports Med 2005;33:1003-10.

18. Arnason A, Andersen TE, Holme l, et al. Prevention of hamstring strains in elite soccer: an intervention study. Scand J Med Sci Sports 2008;18:40-8.

19. Hewett TE, Lindenfeld TN, Riccobene JV, et al. The effect of neuromuscular training on the incidence of knee injury in female athletes. A prospective study. Am J Sports Med 1999;27:699-706.

20. Askling C, Karlsson J, Thorstensson A. Hamstring injury occurrence in elite soccer players after preseason strength training with eccentric overload. Scand J Med Sci Sports 2003;13:244-50.

21. Olsen $\mathbf{0 E}$, Myklebust G, Engebretsen L, et al. Exercises to prevent lower limb injuries in youth sports: cluster randomised controlled trial. BMJ 2005;330:449.

22. McGuine TA, Keene JS. The effect of a balance training program on the risk of ankle sprains in high school athletes. Am J Sports Med 2006;34:1103-11.

23. Pfeiffer RP, Shea KG, Roberts D, et al. Lack of effect of a knee ligament injury prevention program on the incidence of noncontact anterior cruciate ligament injury. J Bone Joint Surg Am 2006;88:1769-74.

24. Emery CA, Rose MS, McAllister JR, et al. A prevention strategy to reduce the incidence of injury in high school basketball: a cluster randomized controlled trial. Clin J Sport Med 2007;17:17-24.

25. Hagglund $\mathbf{M}$, Walden $\mathbf{M}$, Ekstrand J. Lower reinjury rate with a coach-controlled rehabilitation program in amateur male soccer: a randomized controlled trial. Am J Sports Med 2007;35:1433-42.

26. Gilchrist $\mathbf{J}$, Mandelbaum BR, Melancon $\mathrm{H}$, et al. A randomized controlled trial to prevent noncontact anterior cruciate ligament injury in female collegiate soccer players. Am J Sports Med 2008;36:1476-83.
27. Söderman K, Werner S, Pietilä T, et al. Balance board training: prevention of traumatic injuries of the lower extremities in female soccer players? A prospective randomized intervention study. Knee Surg Sports Traumatol Arthrosc 2000;8:356-63.

28. Myklebust G, Engebretsen L, Braekken IH, et al. Prevention of anterior cruciate ligament injuries in female team handball players: a prospective intervention study over three seasons. Clin J Sport Med 2003;13:71-8.

29. Gabbe BJ, Branson R, Bennell KL. A pilot randomised controlled trial of eccentric exercise to prevent hamstring injuries in community-level Australian football. J Sci Med Sport 2006;9:103-9.

30. Engebretsen AH, Myklebust G, Holme l, et al. Prevention of injuries among male soccer players: a prospective, randomized intervention study targeting players with previous injuries or reduced function. Am J Sports Med 2008:36:1052-60.

31. Steffen K, Myklebust G, Olsen OE, et al. Preventing injuries in female youth football - a cluster-randomized controlled trial. Scand J Med Sci Sports 2008;18:605-14

32. Kiani A, Hellquist $\mathrm{E}$, Ahlqvist $\mathrm{K}$, et al. Prevention of soccer-related knee injuries in teenaged girls. Arch Intern Med 2010;170:43-9

33. Soligard T, Myklebust G, Steffen K, et al. Comprehensive warm-up programme to prevent injuries in young female footballers: cluster randomised controlled trial. BMJ 2008;337:a2469.

34. Olsen $\mathbf{0 E}$, Myklebust $\mathrm{G}$, Engebretsen $\mathrm{L}$, et al. Injury pattern in youth team handball: a comparison of two prospective registration methods. Scand J Med Sci Sports 2006;16:426-32.

35. DeVellis RF. Scale development: theory and applications. California, USA: Sage Publications, 2003:1-176.

36. Ajzen I, Fishbein M. Understanding attitudes and predicting social behavior. Englewood Cliffs, New Jersey, USA: Prentice-Hall, 1980:1-278.

37. Bandura A. Self-efficacy. The exercise of control. New York, USA: WH Freeman 1997:1-604.

38. Rivis A, Sheeran P, Armitage CJ. Augmenting the theory of planned behaviour with the prototype/willingness model: predictive validity of actor versus abstainer prototypes for adolescents' health-protective and health-risk intentions. $\mathrm{Br} \mathrm{J}$ Health Psychol 2006;11:483-500.

39. Ajzen I. From intention to actions: a theory of planned behavior. In: Kuhl J Beckmann J, eds. Action-control: from cognition to behavior. Berlin: Springer, 1985:11-39. 